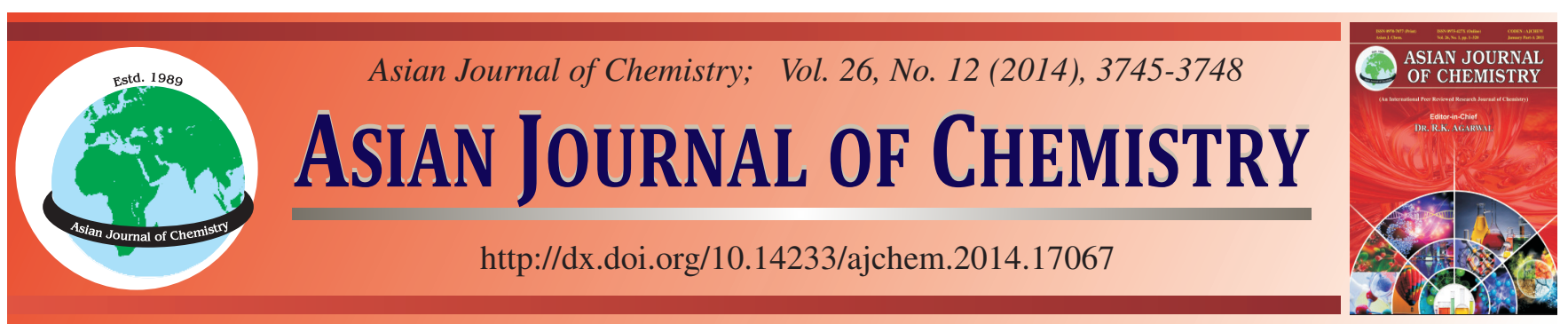

\title{
LC-MSMS and GC-MS Analysis in Identification of Antioxidant and Immuno-Modulatory Molecules in Amukkara curanam $\dagger$
}

\author{
K.P. Arun, V. Sudha and P. BRINDHA*
}

Centre for Advanced Research in Indian System of Medicine, SASTRA University, Thanjavur-613 401, India

*Corresponding author: E-mail: brindha@carism.sastra.edu

\begin{abstract}
Amukkara curanam an anti HIV Siddha formulation was selected for the present work with a view to identify bioactive molecules that might be responsible in improving the immunity in HIV patients. This preparation is often prescribed by Siddha practitioners to manage this disorder. The selected formulation is procured from SKM Siddha Pharmacy (GMP certified), Erode, which contains eight different herbal ingredients. Attempts were being made to identify the bio active constituents present in the selected drug through GC-MS and LCMSMS analysis. This is the first report on the chemical analysis of Amukkara curanam using sophisticated instrumentation techniques. The study revealed presence of antioxidant molecules such as furanone, caryophyllene oxide, $\alpha$-zingiberene, zingiberone, apiol, $\alpha$ eudesmol, nerolidol and gingerol. Besides, piperic acid an antioxidant and anti-viral molecule was also detected. Bio-enhancer and immunomodulatory agents such as piperine, withaferin A and withanolide A were also identified. Present study can contribute in providing chemical evidences for usage of this traditional preparation in the management of HIV, which helps the patients in enhancing their immune system.
\end{abstract}

Keywords: Amukkara curanam, LC-MSMS, GC-MS, Bioactive molecules.

\section{INTRODUCTION}

Herbal medicines are now resorted to as the best alternative system of medicines by the majority of world population. But the greatest lacuna existing in Traditional Indian herbal medicines are validation and standardization. In the present paper, a traditional Siddha preparation Amukkara curanam $^{1}$ which is often prescribed by Siddha practitioners in immune deficiency disorder such as AIDS is selected and evaluated using sophisticated instrumentation techniques such as LC-MSMS and GC-MS to provide supporting chemical evidences for its the rapeutic claims and in determining chemical standards, which can contribute significantly in establishing quality of Siddha preparations and making the system an internationally acceptable and globally recognizable.

Present work deals with developing methods for the chemical standardization of Amukkara curanam, a poly herbal siddha formulations constituting of eight herbal plants. With this view an attempt is also being made to standardize and to confirm the type of active molecules present in the formulation through LC-MSMS and GC-MS analysis.

\section{EXPERIMENTAL}

GC-MS analysis: GC analysis was carried out on a Clarus 500 gas chromatograph using a non-polar, Elite- $5 \mathrm{~ms}$ column (30 $\mathrm{m} \times 0.25 \mathrm{~mm} \times 0.25 \mu \mathrm{m}$ film thickness, coated with $5 \%$ diphenyl-95\% dimethyl polysiloxane) interfaced with mass detector. Helium was used as carrier gas with a flow rate of 1 $\mathrm{mL} / \mathrm{min}$. Temperature programme was $50-150^{\circ} \mathrm{C}$ hold for 2 min at the rate of $3{ }^{\circ} \mathrm{C} / \mathrm{min}$ and increased to $290^{\circ} \mathrm{C}(10 \mathrm{~min})$ at the rate of $8^{\circ} \mathrm{C} / \mathrm{min} .1 \mu \mathrm{L}$ of the methanol extract was injected with split ratio as 1:10. Mass spectra were recorded in the EI mode at $70 \mathrm{eV}$ in a scan range of 40-600. Injector and ion source temperature were maintained at 280 and $200{ }^{\circ} \mathrm{C}$, respectively. The resulted spectrum was compared with NIST library database.

LCMS-MS analysis: Polar and semi polar molecules of aqueous extract of Amukkarac curanam was separated and identified using LC-MSMS. $1 \mathrm{mg} / \mathrm{mL}$ extract was chromatographed over $\mathrm{C}_{18} \mathrm{RP}$ column (Acclaim $120 \AA$, $2.1 \mathrm{~mm} \times 150$ $\mathrm{mm}, 3.0 \mu \mathrm{m}$, Dionex, USA). Eluted compounds were then identified using MS and their respective MSMS pattern. UHPLC was conditioned at $0.2 \mathrm{~mL} / \mathrm{min}$ flow rate, with gradient 


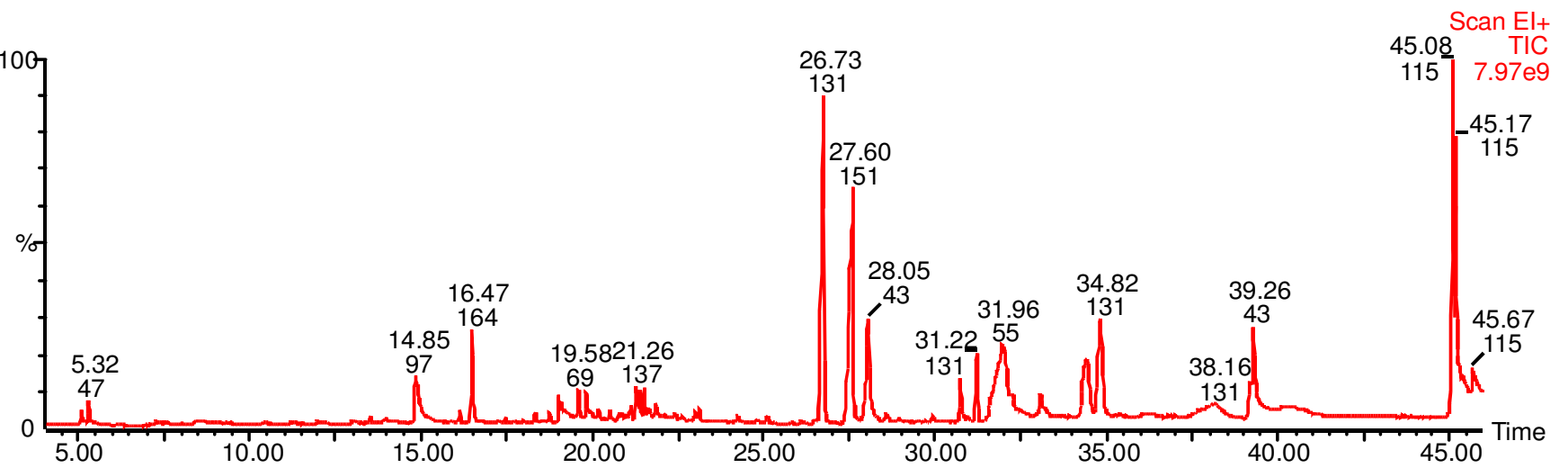

Fig. 1. GC-MS total ion chromatogram of Amukkara curanam

mobile system start at $1 \%$ acetonitrile for $0.2 \mathrm{~min}$ and $99 \%$ water (1\% acetic acid). This was then brought to $75 \%$ acetonitrile at 16 th $\mathrm{min}$ and then reaching at $100 \%$ acetonitrile at 19 th min to $5 \%$ acetonitrile at $21 \mathrm{st}$ min and was maintained at same condition till run ends at 23 rd min. Absorbance was read arbitrary at $325 \mathrm{~nm}$. Exact mass of each eluted compound and their fragmented pattern (MSMS) were identified using ESI-Q-II TOF (Bruker, Germany) at negative mode, nebulizer was set at 30.5 psi with $6.0 \mathrm{~L} / \mathrm{min} \mathrm{N}_{2}$ flow rate. Masses were analyzed in $50-1000 \mathrm{~m} / \mathrm{z}$ range, keeping capillary voltage of $4500 \mathrm{~V}$ with dry heater temperature at $280^{\circ} \mathrm{C}$.

\section{RESULTS AND DISCUSSION}

The ingredients used in the selected Siddha preparation is presented in Table- 1 .

\begin{tabular}{ccc}
\multicolumn{3}{c}{ TABLE-1 } \\
INGREDIENTS OF Amukkarac curanam \\
\hline Plant/drug name & Scientific/common name & Quantity (g) \\
\hline Nattu Amukkara. Kizhangu & Withania somnifera & 640 \\
Cukku & Zingiber officinale & 320 \\
Tippili & Piper nigrum & 160 \\
Milaku & Piper longum & 80 \\
Elakkai & Eletteria cardamom & 40 \\
Sirunagapoo & Mesuafera & 20 \\
Kirambu & Syzygium aromaticum & 10 \\
Carkkarai & Saccharum officinarum & 1280 \\
\hline
\end{tabular}

GC-MS analysis: GCMS data presented in Table-2 shows the presence of 26 fragmented compounds of which phenols, flavonoid and sugar moieties were found to be higher. The fragmented pattern of different compounds present in the Amukkarac curanam were detected and identified based on their fragment pattern available in NIST library. Furanone ${ }^{2}$, caryophyllene oxide ${ }^{2}, \alpha$-zingiberene ${ }^{3}$, apiol ${ }^{4}, \alpha$-eudesmol ${ }^{5}$, nerolidol $^{6}$, gingerol ${ }^{7}$ and piperic acid $^{8}$ are the important bioactive molecules identified and they are scientifically proven for their antioxidant potentials. GC-MS chromatogram of the methanolic extract was shown in Fig. 1 and their respective structures were shown in Fig. 2.

LC-MSMS analysis: LC-MSMS analysis helped in identifying glycowithanolides (Fig. 3) such as withanolide A and steroidal lactones like withaferins $\mathrm{A}$, which are proven immuno modulatory and antioxidant agents ${ }^{9,10}$. Piperine an alkaloid with rich bioenhancing and antioxidant potential was also identified ${ }^{11,12}$.

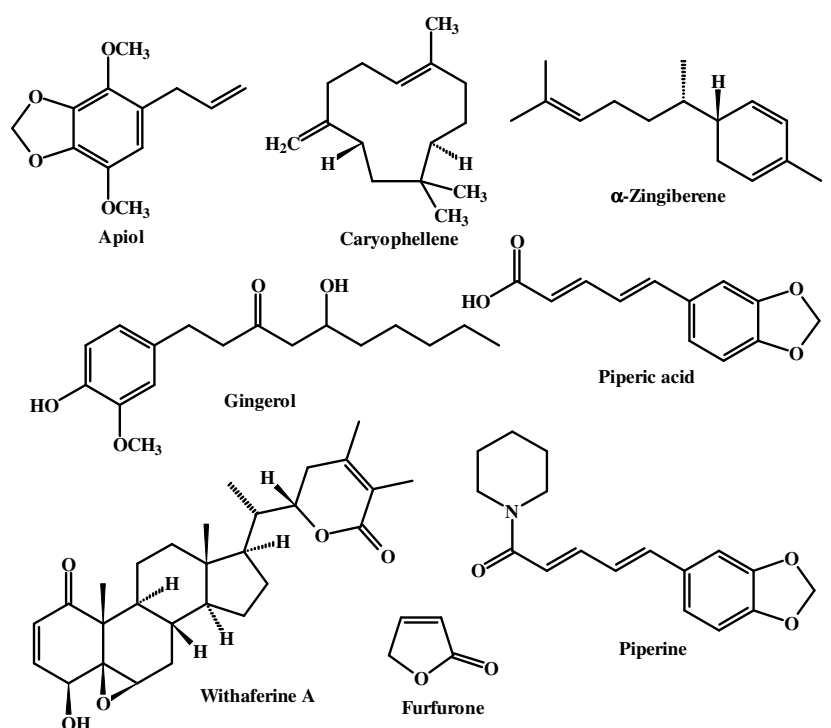

Fig. 2. Various antioxidant molecules identified in Amukkara curanam through GC-MS
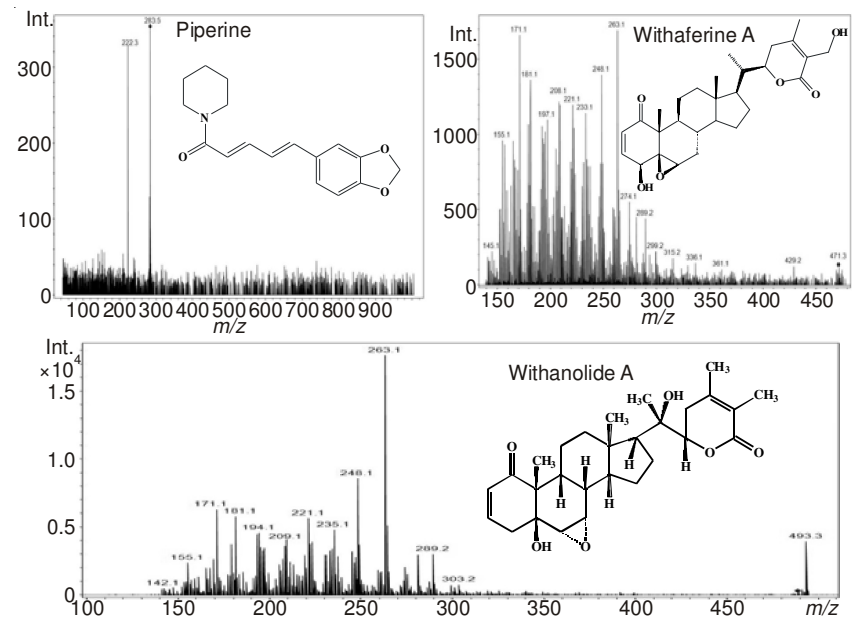

Fig. 3. MSMS fragmentation pattern of different phytoconstituents present in Amukkarac curanam

\section{Conclusion}

From the study it is concluded that major chemical constituents identified must have been derived from the major plant ingredients used in the formulation such as withanolides and withaferins from Withania somnifera, zingiberene and 


\begin{tabular}{|c|c|c|c|}
\hline \multicolumn{4}{|l|}{$\begin{array}{c}\text { TABLE-2 } \\
\text { LIST OF FRAGMENTED COMPOUNDS }\end{array}$} \\
\hline Peak name & Retention time & Peak area & Peak area $(\%)$ \\
\hline Name: Hexane, 1-(ethenyloxy), m.f.: $\mathrm{C}_{8} \mathrm{H}_{16} \mathrm{O}$, m.w.: 128 & 5.09 & 14937520 & 0.3917 \\
\hline Name: Furfural, m.f.: $\mathrm{C}_{5} \mathrm{H}_{4} \mathrm{O} 2$, m.w.: 96 & 6.11 & 3511483 & 0.0921 \\
\hline Name: 4,5-Dihydro-2-methylimidazole-4-one, m.f.: $\mathrm{C}_{4} \mathrm{H}_{6} \mathrm{~N}_{2} \mathrm{O}$, m.w.: 98 & 8.45 & 27283022 & 0.7154 \\
\hline Name: 4-Octanol, 7-methyl-, m.f.: $\mathrm{C}_{9} \mathrm{H}_{20} \mathrm{O}$, m.w.: 144 & 9.12 & 2023926 & 0.0531 \\
\hline Name: Octanal, m.f.: $\mathrm{C}_{8} \mathrm{H}_{16} \mathrm{O}$, m.w.: 128 & 9.42 & 1774075 & 0.0465 \\
\hline Name: 2,5-Dimethyl-4-hydroxy-3(2H)-furanone, m.f.: $\mathrm{C}_{6} \mathrm{H}_{8} \mathrm{O}_{3}$, m.w.: 128 & 11.53 & 891244 & 0.0234 \\
\hline Name: Phenylethyl alcohol, m.f.: $\mathrm{C}_{8} \mathrm{H}_{10} \mathrm{O}$, m.w.: 122 & 12.21 & 1071588 & 0.0281 \\
\hline Name: 4H-Pyran-4-one, 2,3-dihydro-3,5-dihydroxy-6-methyl-, m.f.: $\mathrm{C}_{6} \mathrm{H}_{8} \mathrm{O}_{4}$, m.w.: 144 & 13.02 & 10391134 & 0.2725 \\
\hline Name: Decanal, m.f.: $\mathrm{C}_{10} \mathrm{H}_{20} \mathrm{O}$, m.w.: 156 & 13.49 & 7272088 & 0.1907 \\
\hline Name: Glycerin, m.f.: $\mathrm{C}_{3} \mathrm{H}_{8} \mathrm{O}_{3}$, m.w.: 92 & 14.01 & 13342372 & 0.3499 \\
\hline Name: 2-Furancarboxaldehyde, 5-(hydroxymethyl)-, m.f.: $\mathrm{C}_{6} \mathrm{H}_{6} \mathrm{O}_{3}$, m.w.: 126 & 14.85 & 107761416 & 2.8257 \\
\hline Name: 2-Methoxy-4-vinylphenol, m.f.: $\mathrm{C}_{9} \mathrm{H}_{10} \mathrm{O}_{2}$, m.w.: 150 & 15.88 & 2928621 & 0.0768 \\
\hline Name: 3-Cyclohexene-1-methanol, $\alpha, \alpha, 4$-trimethyl-, acetate, m.f.: $\mathrm{C}_{12} \mathrm{H}_{20} \mathrm{O}_{2}$, m.w.: 196 & 16.11 & 10476081 & 0.2747 \\
\hline Name: Phenol, 2-methoxy-3-(2-propenyl)-, m.f.: $\mathrm{C}_{10} \mathrm{H}_{12} \mathrm{O}_{2}$, m.w.: 164 & 16.47 & 114481488 & 3.0019 \\
\hline Name: 9-Octadecene, (E)-, m.f.: $\mathrm{C}_{18} \mathrm{H}_{36}$, m.w.: 252 & 16.66 & 1044930 & 0.0274 \\
\hline Name: $n$-Decanoic acid, m.f.: $\mathrm{C}_{10} \mathrm{H}_{20} \mathrm{O}_{2}$, m.w.: 172 & 16.74 & 2385572 & 0.0626 \\
\hline Name: Caryophyllene, m.f.: $\mathrm{C}_{15} \mathrm{H}_{24}$, m.w.: 204 & 17.48 & 5017894 & 0.1316 \\
\hline Name: Benzaldehyde, 3-hydroxy-4-methoxy-, m.f.: $\mathrm{C}_{8} \mathrm{H}_{8} \mathrm{O}_{3}$, m.w.: 152 & 17.72 & 3311743 & 0.0868 \\
\hline $\begin{array}{l}\text { Name: 2-Propenoic acid, 2-methyl-, 3-methyl-2-methylene-3-butenyl ester, m.f.: } \mathrm{Cl}_{0} \mathrm{H}_{14} \mathrm{O}_{2} \text {, } \\
\text { m.w.: } 166\end{array}$ & 18.00 & 1172008 & 0.0307 \\
\hline Name: Benzene, 1-(1,5-dimethyl-4-hexenyl)-4-methyl-, m.f.: $\mathrm{C}_{15} \mathrm{H}_{22}$, m.w.: 202 & 18.33 & 7655299 & 0.2007 \\
\hline$\alpha$-Zingiberene, m.f.: $\mathrm{C}_{15} \mathrm{H}_{24}$, m.w.: 204 & 18.52 & 4386292 & 0.1150 \\
\hline Myristicin, m.f.: $\mathrm{C}_{11} \mathrm{H}_{12} \mathrm{O}_{3}$, m.w.: 192 & 18.77 & 10321045 & 0.2706 \\
\hline Name: Phenol, 2,4-bis(1,1-dimethylethyl)-, m.f.: $\mathrm{C}_{14} \mathrm{H}_{22} \mathrm{O}$, m.w.: 206 & 19.01 & 19329832 & 0.5069 \\
\hline Name: $\tau$-Cadinol, m.f.: $\mathrm{C}_{15} \mathrm{H}_{26} \mathrm{O}$, m.w.: 222 & 19.09 & 3925454 & 0.1029 \\
\hline Name: Elemol, m.f.: $\mathrm{C}_{15} \mathrm{H}_{26} \mathrm{O}$, m.w.: 222 & 19.58 & 28615998 & 0.7504 \\
\hline Name: Diepi- $\alpha$-cedrene epoxide, m.f.: $\mathrm{C}_{15} \mathrm{H}_{24} \mathrm{O}$, m.w.: 220 & 20.15 & 890574 & 0.0234 \\
\hline Name: Caryophyllene oxide, m.f.: $\mathrm{C}_{15} \mathrm{H}_{24} \mathrm{O}$, m.w.: 220 & 20.19 & 370887 & 0.0097 \\
\hline Name: $\alpha$-Bisabolol, m.f.: $\mathrm{C}_{15} \mathrm{H}_{26} \mathrm{O}$, m.w.: 222 & 20.49 & 7345865 & 0.1926 \\
\hline .Zingiberone, m.f.: $\mathrm{C}_{11} \mathrm{H}_{14} \mathrm{O}_{3}$, m.w.: 194 & 21.26 & 17343860 & 0.4548 \\
\hline Name: Apiol, m.f.: $\mathrm{C}_{12} \mathrm{H}_{14} \mathrm{O}_{4}$, m.w.: 222 & 21.51 & 21852672 & 0.5730 \\
\hline Name: 6-Octen-1-yn-3-ol, 3,7-dimethyl-, m.f.: $\mathrm{C}_{10} \mathrm{H}_{16} \mathrm{O}$, m.w.: 152 & 21.64 & 5584531 & 0.1464 \\
\hline Name: 6,10-Dodecadien-1-yn-3-ol, 3,7,11-trimethyl-, m.f.: $\mathrm{C}_{15} \mathrm{H}_{24} \mathrm{O}$, m.w.: 220 & 21.84 & 13838941 & 0.3629 \\
\hline Name: N-Isobutyl-(2E,4Z)-octadienamide, m.f.: $\mathrm{C}_{12} \mathrm{H}_{21} \mathrm{NO}$, m.w.: 195 & 22.38 & 8447631 & 0.2215 \\
\hline Name: Tetradecanoic acid, m.f.: $\mathrm{C}_{14} \mathrm{H}_{28} \mathrm{O}_{2}$, m.w.: 228 & 23.01 & 4389323 & 0.1151 \\
\hline Name: 3-Eicosene, (E)-, m.f.: $\mathrm{C}_{20} \mathrm{H}_{40}$, m.w.: 280 & 23.11 & 5826440 & 0.1528 \\
\hline Name: 3,7,11,15-Tetramethyl-2-hexadecen-1-ol, m.f.: $\mathrm{C}_{20} \mathrm{H}_{40} \mathrm{O}$, m.w.: 296 & 24.06 & 2775829 & 0.0728 \\
\hline (+)- $\alpha$-Bisabolol, m.f.: $\mathrm{C}_{15} \mathrm{H}_{26} \mathrm{O}$, m.w.: 222 & 24.24 & 15606878 & 0.4092 \\
\hline$\alpha$-Eudesmol, m.f.: $\mathrm{C}_{15} \mathrm{H}_{26} \mathrm{O}$, m.w.: 222 & 24.79 & 8483221 & 0.2224 \\
\hline Cinnamic acid, methyl ester, (E)-, m.f.: $\mathrm{C}_{10} \mathrm{H}_{10} \mathrm{O}_{2}$, m.w.: 162 & 26.73 & 607957120 & 15.9416 \\
\hline $\begin{array}{l}\text { Name: 5-Isopropyl-2,8-dimethyl-9-oxatricyclo[4.4.0.0(2,8)]decan-7-one, m.f.: } \mathrm{C}_{14} \mathrm{H}_{22} \mathrm{O}_{2} \text {, } \\
\text { m.w.: } 222\end{array}$ & 27.60 & 739981632 & 19.4035 \\
\hline Name: $n$-Hexadecanoic acid, m.f.: $\mathrm{C}_{16} \mathrm{H}_{32} \mathrm{O}_{2}$, m.w.: 256 & 28.05 & 319181504 & 8.3695 \\
\hline Name: Phenol, 2-cyclohexyl-, m.f.: $\mathrm{C}_{12} \mathrm{H}_{16} \mathrm{O}$, m.w.: 176 & 28.59 & 13904054 & 0.3646 \\
\hline .Nerolidol, m.f.: $\mathrm{C}_{15} \mathrm{H}_{26} \mathrm{O}$, m.w.: 222 & 28.96 & 6318975 & 0.1657 \\
\hline Name: Naphthalene, decahydro-1,1-dimethyl-, m.f.: $\mathrm{C}_{12} \mathrm{H}_{22}$, m.w.: 166 & 29.91 & 14423179 & 0.3782 \\
\hline Piperic acid, m.f.: $\mathrm{C}_{12} \mathrm{H}_{10} \mathrm{O}_{4}$, m.w.: 218 & 30.74 & 79137096 & 2.0751 \\
\hline Name: 2-Propenamide, N-octyl-3-phenyl-, m.f.: $\mathrm{C}_{17} \mathrm{H}_{25} \mathrm{NO}$, m.w.: 259 & 31.22 & 92154048 & 2.4164 \\
\hline Name: Z,E-3,13-Octadecadien-1-ol, m.f.: $\mathrm{C}_{18} \mathrm{H}_{34} \mathrm{O}$, m.w.: 266 & 31.96 & 410132608 & 10.7544 \\
\hline Name: Gingerol, m.f.: $\mathrm{C}_{17} \mathrm{H}_{26} \mathrm{O}_{4}$, m.w.: 294 & 33.07 & 32617946 & 0.8553 \\
\hline Name: Gingerol, m.f.: $\mathrm{C}_{17} \mathrm{H}_{26} \mathrm{O}_{4}$, m.w.: 294 & 34.42 & 252434752 & 6.6193 \\
\hline Name: 2-(3,4-Methylenedioxyphenyl)cyclohexanone, m.f.: $\mathrm{C}_{13} \mathrm{H}_{14} \mathrm{O}_{3}$, m.w.: 218 & 34.82 & 280951136 & 7.3670 \\
\hline Name: 2-Propenamide, N-dodecyl-3-phenyl-, m.f.: $\mathrm{C}_{21} \mathrm{H}_{33} \mathrm{NO}$, m.w.: 315 & 38.16 & 90135288 & 2.3635 \\
\hline Name: Lanost-8-en-3-ol, $(3 \alpha)-$, m.f.: $\mathrm{C}_{30} \mathrm{H}_{52} \mathrm{O}$, m.w.: 428 & 39.26 & 55009816 & 1.4424 \\
\hline Name: Piperine, m.f.: $\mathrm{C}_{17} \mathrm{H}_{19} \mathrm{NO}_{3}$, m.w.: 285 & 45.08 & 301235584 & 7.8989 \\
\hline
\end{tabular}

other aromatic compounds from Zingiber officinalis and piperine from Piper species. These identified molecules are proven antioxidants and immuno-modulatory agents. It is inferred that this formulation must be working in HIV/AIDS patients probably through improving their antioxidant status and boosting their immune system.

\section{ACKNOWLEDGEMENTS}

The authors are thankful to Vice-Chancellor of SASTRA University, Thanjavur for utilizing the facilities to carry out the research work and Indian Council of Medical Research (ICMR), New Delhi for funding (No. 61/6-3/2007-HIV/DMS/ TRM Dt. 29.05.08). 


\section{REFERENCES}

1. Anonymous, The Siddha Formulary of India Part-I, edn 1, Ministry of Health and Family Welfare, New Delhi, p. 152 (1979).

2. B. Kaurinovic, S. Vlaisavljevic, M. Popovic, D. Vastag and M. DjurendicBrenesel, Molecules, 15, 5943 (2010).

3. S. Banerjee, H.I. Mullick and J. Banerjee, Int. J. Pharm. Biol. Sci., 2, 283 (2011)

4. I. Elisia and D.D. Kitts, The Antioxidant Potential of Parsley and its Constituents; CAB Reviews: Perspectives in Agriculture, Veterinary Science, Nutrition and Natural Resources, Nutrition and Natural Resources, 3, 1 (2009)

5. S. H. Kim, S. Y. Lee, C. Y. Hong, K. S. Gwak, M. J. Park, D. Smith, I. G. Choi, Int. J. Cosmet. Sci., 35, 484 (2013).

6. F. Amezouar, W. Badri, M. Hsaine, N. Bourhim and H. Fougrach, J. Appl. Pharm. Sci., 2, 212 (2012).
7. Y. Masuda, H. Kikuzaki, M. Hisamoto and N. Nakatani, Biofactors, 21, 293 (2004).

8. J. Upadhyay, R.K. Kesharwani and K. Misra, Bioinformation, 4, 233 (2009).

9. R. Mittal and R.L. Gupta, Methods Find Exp. Clin. Pharmacol., 22, 271 (2000).

10. S.K. Bhattacharya, K.S. Satyan, S. Ghosal, Indian J. Exp. Biol., 35, 236 (1997).

11. S.K. Verma and A. Kumar, Asian J. Pharm. Clin. Res., 4, S1 (2011).

12. K.E. Heim, A.R. Tagliaferro and D.J. Bobilya, J. Nutr. Biochem., 13, 572 (2002)

13. P. Akbay, A.A. Basaran, U. Undeger and N. Basaran, Phytother. Res., 17, 34 (2003)

14. E.J. Middleton, Adv. Exp. Med. Biol., 439, 175 (1998). 